\title{
SOME OBSERVATIONS ON THE ROLE OF ALLERGY IN LEPROSY (2).
}

\section{PART II.-ALLERGY AND THE MACULAR SERIES.}

T. F. DAVEY.

It is customary to describe three varieties of leprous macule. The Cairo Conference (1938) contented itself with two, recognising (a) Simple Macular (with flat macules), and Tuberculoid Macular (with major and minor subtypes), subvarieties of neural leprosy, but admitting that the term, Macule, " is sometimes used to designate lepromatous patches." Lowe, (1936) and Rogers and Muir (1946) all describe and illustrate a lepromatous form of macule sufficiently distinct to merit separate classification.

Macules are the commonest feature of leprosy in Nigeria. Their great diversity, while clearly indicating their immunological importance, is at the same time an obstacle to their effective classitication. An understanding of their role in the natural history of the disease and their consequent classification is eminently desirable, for macules undoubtedly form the link between the immune individual on the one hand, in whom no visible disease appears, and the highly susceptible person on the other hand, who exhibits anergic lepromatous leprosy. The three recognised varieties, (a) lepromatous, (b) simple neural (flat) macules, and (c) tuberculoid macules, do not provide an acceptable classification of macular leprosy for at least two reasons. In the first place, the distinction made between tuberculoid macules and simple neural flat macules is unsound, for it throws the emphasis on the degree of thickening and elevation of a macule as its distinguishing feature. All types of macule represent a localised reaction to the lepra bacillus in the skin, the effectiveness of which is to be measured not by the degree of thickening and elevation in the macule, but rather by the success with which the bacillus is localised and destroyed. This may certainly occur effectively in a flat macule. Wade, (1937) and Ermakova, (1939) have clearly demonstrated by extensive histological studies that pale flat macules are in many cases intimately related to clinically tuberculoid macules, the difference being one of degree only. In such cases the disease is confined mainly to the papillary layer of the corium, and though epithelioid cells rarely form actual tubercles, their presence conclusively indicates the allergic nature of the response, and its unity with that occurring in tuberculoid macules. 
It has been pointed out in Part I (Davey 1946) that the allergic tuberculoid response may be effective over a considerable range of intensity. The minor tuberculoid macule may in appropriate circumstances represent as effective a response as does the more violent major tuberculoid response. In the same way the even milder response which appears to the naked eye as a flat macule may be adequate in favourable circumstances.

An even more fundamental weakness in the triple classification is its inadequacy. Although in their typical form the three varieties represent readily identifiable types of macule, deviations from type are so numerous in clinical practice that it is imjossible to place all macules in these three compartments without broadening the description of each to such an extent that much of their value is lost. There are more than three types of leprous macule, and the three standard varieties cannot therefore embrace them.

\section{The Macular Series.}

If the macule indicates a reaction to the bacillus designed to localise and destroy it, the only reasonable criterion for classifying macules of varying appearance is the degree of success with which this fulfils its function. The thickening of the skin in the macule is a secondary consideration, as is also the number of bacilli found on a single examination. What matters is the speed with which the bacilli are destroyed, the sterilising power of the response. If a sufficiently large number of cases of macular leprosy is examined and observed over a period, it is possible to arrange the various macular forms in descending order of effectiveness, and if this is done it is apparent that they do not fall into a few, well defined groups, but rather form a continuous series. At one end of the scale are seen the effective, highly localising macules typical of the most advantageous types of tuberculoid and pale flat macule. At the other end are seen the innumerable poorly defined macules of the least satisfactory lepromatous group in which there is no effective localisation and the macules represent a temporary phase in the advance of the infection. Between these two extremes are seen macular forms, through the increasing deviations of which the gulf between the two extremes is effectively bridged and a continuous series produced with no distinct break at any point. Before discussing the implications of this series, it is perhaps desirable to describe it in greater detail.

\section{(a) The Optimal Allergic Group.}

The two extremes of the series are well defined. At the 
most effective end of the scale are placed macules, clinically tuberculoid or simple neural, having the following characteristics.

1. The number single or multiple.

2. Sharp definition of the edge, only consistent with a relatively slow rate of spread.

3. Rapid destruction of bacilli in the lesion to produce a negative bacteriological test.

4. Spontaneous resolution, which may occur simultaneously through the lesion, but more commonly appears as a spreading zone of healing in the centre of the macule.

5. Hypopigmentation of varying degree in dark skins, which may or may not be associated with erythema.

6. Interference with local nerve function, leading to loss of sensation in the macule.

7. A positive lepromin test.

These characteristics may be seen in macules of all degrees of thickening and elevation, and all macules exhibiting them, whether they are raised or flat, should be grouped together into a single optimal allergic group.

Among these characteristics, rapid destruction of bacilli is the most fundamental, but is difficult to demonstrate in its later stages, as a persistently negative bacteriological test is still consistent with a macule which continues to spread. The standard bacteriological test is a gross method of investigation. Spontaneous resolution is perhaps the feature of greatest practical value, and no case should be classified in this group unless it is in evidence. Loss of nerve function in the skin of the macule is generally believed to be the mechanical result of the cellular reaction in the cutaneous nerve, caused by ascending infection. Some infections are more neurophilic than others, but in general the degree of nerve involvement runs more or less parallel with the intensity of the response, being most pronounced in major tuberculoid lesions. A certain amount of time is required for pressure effects to manifest themselves, so that sensation may be normal in the early stages of macules. 'Loss of thermal sensation, of light touch, and sensibility to pain usually advance in that order. Loss of sensation is frequently absent in macules on the face. Loss of nerve function is thus not an invariable accompaniment of macules in this group.

Within the optimal allergic group, the most advantageous form of macule is undoubtedly the single tuberculoid macule, and this in many instances represents a purely localised infection, 
destined to be controlled and eliminated. In abortive infections this local lesion may be small and insignificant, and not recognised as leprosy by the patient. Subclinical cases of this type are frequently seen on leprosy surveys among the contacts of more advanced cases.

\section{(b) Lepromatous Macules.}

At the opposite end of the scale are found lepromatous macules, which stand in sharp contrast to macules of the optimal allergic group, and have the following characteristics.

1. They are usually extremely numerous and, if few when first seen, are liable to multiply and become numerous.

2. The edge is poorly defined and macules rapidly spread and coalesce, often to the point of covering the entire body surface.

3. Bacilli are numerous and persistent in the macules, and the skin elsewhere is frequently bacteriologically positive.

t. There is no sign of spontaneous resolution in the centre of macules.

5. Hypopigmentation and erythema are present in varying degree.

6. Nerve involvement is either slight or entirely absent.

7. The lepromin test in normal skin is usually negative.

This group is only one step removed from the diffuse infiltrating and nodular forms of leproma, but is distinguished from these forms by the presence of hypopigmentation combined with the focal arrangement of the lesions. Nodular and diffuse lepromatous ?eprosy may exist even in an advanced state without any sign whatever of hypopigmentation in the form of nacules, though in its later stages there is occasionally seen a vague diffuse pallor, not localised into the form of macules. Lepromatous macules appear at a much earlier stage than this, and are sometimes described as, "Prelepromatous." This is only part of the truth. Sometimes such macules spread and coalesce to form extensive pale areas in which the disease advances to become diffuse leproma, and if the entire body surface is involved, the patient may be thought to be merely a pale skinned individual suffering from uncomplicated anergic lepromatous leprosy, the macular phase being entirely masked. Before this stage is reached, diminishing islands of darker skin, frequently most persistent in the groins, indicate the macular origin of the hypopigmentation. The writer has described a case of this type (Davey, 1942). 
Although the advance of lepromatous macules to diffuse leproma is common, it is by no means invariable. The two types not inf requently coexist in the same patient, but we have seen cases in which the progress of the disease in the macular areas has been halted, both before and after the point where widespread coalescence is followed by a long stationary phase of the disease, with no advance to graver forms, but a gradual decline in the concentration of bacilli in the skin. This welcome state of affairs is only very rarely seen in true anergic diffuse leproma, and even then is only likely in the later stages of the disease, by which time the nose, larynx and testicles have usually become affected.

Lepromatous macules thus represent a slightly more advantageous form of the disease than true diffuse leproma, and though the advantage may be temporary, it docs represent a feeble attempt at localisation in the skin, and is thus distinct from true anergic leprosy.

\section{(c) Intermediate formis.}

Within the two extreme forms there are many intermediate varieties of macule. Working along the series from the optimal group, the first unsatisfactory deviation is displayed by macules. clinically of tuberculoid or simple neural type, but in which spontaneous resolution is delayed or lacking, the macule taking a very chronic course. In the next deviation, deficiency in spontaneous resolution is accompanied by a lack of sharp definition in the edge of the macule. "Streaming" edges are seen, and there is a tendency for adjacent macules to meet and coalesce without this being succeeded by resolution. As these deviations become increasingly pronounced, the number of macules present tends to increase, and further along the series they are usually numerous. At the same time, signs of nerve involvement diminish. We thus arrive at a group exhibiting numerous macules in which there is defective resolution, which have a fairly well defined edge but tend to coalesce, which exhibit only slight signs of nerve involvement, yet remain persistently bacteriologically negative. They thus lie in an intermediate position between the extreme forms. Macules of this type are common in Nigeria, and both flat and raised forms exist. A distinct variety takes the form of innumerable papules which spread and flatten to form small hypopigmented macules which then proceed to coalesce, the bacteriological test remaining negative. The Cairo classification makes no provision for a case of this sort. From these intermediate cases it is a short step to macules resembling lepromatous macules, yet still persistently bacteriologically negative, until 
through similar lesions with increasing bacillary content we finally arrive at typical lepromatous macules.

Reviewing the series as a whole, there is seen a progressive loss of definition and resolution in macules towards the lepromatous end, accompanied by an increase in the persistent bacillary content. Features common to all types of macules are hypopigmentation and erythema, both of which are variable. Sensory changes in the macule also decline and disappear towards the lepromatous end, though they may still be in evidence apart from macules.

The macular series can be traced most perfectly where the macules are flat or of mild elevation. Nevertheless it is of interest to note that unfavourable deviations occur even with major tuberculoid macules. Cases of this type have been described by Warle (1940).

\section{Implications of the Macular Series.}

The very existence of the macular series has important implications. The absence of any distinct break in it indicates that the difference between one form and another is not due to fundamentally distinct disease processes, but is quantitative rather than qualitative, produced by varying degrees of the same factors, rather than entirely different factors. In other words, that although appearances differ, there is a common thread running through the entire series and binding one member to the next. It is generally rcognised that tuberculoid leprosy has an allergic basis, and the clinical appearance of the tuberculoid macule is therefore the resultant of three varying factors, (a) antigen concentration, (b) local antibody production, and (c) the allergic cellular reaction to the antigen-antibody complex. It is reasonable to consider the entire series as produced by the interplay of these same factors in varying intensity, increasingly unsatisfactory forms of macule being manifest as the local allergic defence mechanism declines in effectiveness in relation to antigen concentration. If this is the case, we are witnessing along the macular series the progressive failure of the allergic response, from optimal at the tuberculoid end, to transitory and ineffective at the lepromatous end.

There is considerable evidence, both clinical and experimental, to support the conclusion that an allergic mechanism underlies all macular forms of leprosy, whatever other factors may be involved. From the clinical standpoint, (a) it is difficult to explain the sudden appearance of a crop of macules apart from allergy. Such a crop is a very common occurrence and the maçules may conform not only to the optimal group, but to any 
point in the macular series. (b) The sudden appearance of a macule $\frac{1}{2}$ inch or more in diameter is best explained on a basis of allergy. (c) The existence of the macular series itself is best explained in the same way. From the experimental point of view, two methods of investigation are open to us, (a) histological studies, (b) the lepromin test. At the present time, thorough histological studies have been made only of the optimal group of macules, and studies of the intermediate forms and the lepromatous group are inadequate for judgments to be made. The lepromin test forms a simple and ready means of investigation, and has been applied to macules of various types.

\section{The Lepromin Test and the Macular Series.}

A positive lepromin test in an infected person indicates the existence of allergic hypersensitiveness to the antigens of the lepra bacillus. A positive test is a commonplace in tuberculoid leprosy, but in order to investigate the status of other macular forms we have applied the test to some hundreds of patients among whom all varieties of macular leprosy are represented. In view of the important local aspect of allergy in leprosy, discussed in Part I, and in order to discover the allergic state of macules, the test has been carried out in the macules themselves as well as in normal skin.

For the purposes of this work, three types of lepromin were used, (a) crude lepromin prepared locally by the method described by Rogers and Muir (1940), (b) partially purified lepromin prepared locally, following the earlier stages of Dharmendra's technique (1941), (c) samples of Dharmendra's purified lepromin kindly supplied by the courtesy of Dr. E. Muir.

It was first necessary to compare the three types of lepromin used. For this purpose, 14 patients were selected, all exhibiting tuberculoid macules in a state of activity. Adjacent or comparable macular sites were injected simultaneously with $0.1 \mathrm{cc}$. of two of the three types of lepromin and observed for 48 hours. From a study of the early reactions produced, it was found that the three types were of approximately equal activity, the locally prepared crude lepromin giving at least as powerful a reaction as the purified varieties. As supplies of the latter were limited the same sample of crude lepromin was used in the majority of patients submitted to later tests.

For the purpose of these tests, patients were divided into six groups according to the type of macule they exhibited, as follows.

Group 1. Optimal allergic macules, well defined tuberculoid or 
simple neural macules, exhibitings spontaneous resolution, nerve involvement usually present.

Group 2. Macules well defined, but exhibiting deficient resolution.

Group 3. Macules showing deficiency of resolution and also lack of definition, tending to spread and coalesce.

Group 4. Macules numerous and discrete, resolution defective, nerve involvement slight or lacking, tendency in the macules to coalesce, bacteriologically negative.

Group 5. Macules very numerous, coalescent, nerve involvement rare, no resolution, bacilli scanty.

Group 6. Lepromatous macules. Very nurnerous, definition poor, coalescent, nerve involvement rare, bacilli numerous and persistent.

In each group, cases with raised and flat types of macule were sought.

In carrying out the tests, $0.1 \mathrm{cc}$. of one or other type of lepromin was injected intradermally into the skin of the inacule. near to an active edge, and another $0.1 \mathrm{cc}$. was injected into normal skin. For purposes of control, an extract of skin obtained by biopsy from a non-leprous individual was used, and prepared by the Rogers and Muir method in a manner identical with that used for lepromin. $0.1 \mathrm{cc}$. of this was injected into the macule and into the normal skin, simultaneously with the lepromin, in the first 70 patients tested. Although slight reaction to this extract were obtained in some patients, it was found that this logical control to the lepromin tests in no way impaired the specificity of the latter. Readings were made after 24 and 48 hours, and in some patients after 32 hours. It was found that in general the early reaction was most marked after 24 to 32 hours, and towards the end of the series, when working with out-patients, the 48 hour reading was abandoned.

No special attempt was made to test a large number of patients in group 1, as a positive result was to be expected in all patients in this group. The numbers tested in other groups vary widely, as some types of macule are seen much more commonly than others.

RESULTS.

\section{TABLE I.}

Group 1. Macules of optimal tuberculoid type.

(a) Raised Macules.

Macules

No. tested. Strongly pos. Positive. Doubtful. Negative

Normal skin

$23 \quad 5$


(b) Flat Macules.

Very few macuies of this type werc tested and results are not regarded as significant.

Group 2. Macules deficient in spontaneous resolution, but well defined.

(a) Ruised Macules.

No. tested. Strongly pos. Positive. Doubtful. Negative

Macules $\quad$........... 30

Normal skin ...... 30

13

$\begin{array}{ll}3 & 7\end{array}$

(b) Flat Macules.

No. tested. Strongly pos. Positive. Doubtful. Negative

Macules $\quad$............ 10

Normal skin ...... 10

$\begin{array}{lll}3 & 1 & 1 \\ 9 & 1 & 1\end{array}$

Group 3. Macules showing deficiency of resolution and also lack of definition, tending to spread and coalesce.

(a) Raised Mucules.

No. tested. Strongly pos. Positive. Doubtful. Negative

Macules

$\begin{array}{rrrrr}68 & 34 & 27 & 6 & 1 \\ 67 & 8 & 30 & 17 & 12\end{array}$

Normal skin ...... 67

(b) Filat Macules.

No. tested. Strongly pos. Positive. Doubtful. Negative

Macules 39

Normal skin ...... 39

$14 \quad 21$

21

10

4

Grour 4.

39

2

$13 \quad 10$

14 involvement slight or lacking, tendency to coalesce, bacteriologicaliy negative.

(a) Raiscd Macules.

No. tested. Strongly pos. Positive. Doubtfu!. Negative

Vacules

31

Normal skin ...... 29

18

7

8

4

11

2

(b) Fiat Macules.

No. tested. Strongly pos. Positive. Doubtful. Negative

M:cules 42

Normal skin ...... 42

18

14

11

17

14

Group 5. Macules numerous, coalescent, nerve invclvement rire, no resolution, bacilli persistent but scanty.

(a) Raised Macules.

No. tested. Strongly pos. Positive. Doubtful. Negative

Macules 34

Normal skin ...... 34 $1 ?$

8

5
11

13

(b) Flat Macules.

No. tested. Strongly pos. Positive. Doubtful. Negative

Macules 35

Normal skin ...... 35

17

8

11

13

4

Group 6. Leprumatous macules. Very nume:ous, definition poor, lopromatous, bacilli numerous and persistent.

(a) Raised Macules.

No. tested. Strongly pos. Positive. Doubtful. Negative

Macules

$$
40
$$

Normal skin ...... 35

12 22

9

4

13

2

(b) Flat Macules.

No. tested. Strongly pos. Positive. Doubtful. Negative

Macules

22

Normal skin ...... 22

2

8

6

6 
In order to simplify comparisons between one group and ancther, these results are given as percentages of the total in each group in the following tables.

TABLE II. Lepromin results in patients with raised macules.

(a) Tests in Macules.

Group. Strongly positive. Positive. Doubtful. Negative

$\begin{array}{ccccc} & \% & \% & \% & \% \\ 1 & 57 & 30 & 13 & \text { nil } \\ 2 & 47 & 43 & 10 & \text { nil } \\ 3 & 50 & 40 & 9 & 1 \\ 4 & 58 & 23 & 13 & 6 \\ 5 & 24 & 53 & 14 & 9 \\ 6 & 30 & 55 & 10 & 5\end{array}$

(b) Tests in onormal skin.

Group. Strongly positive. Positive.

$\begin{array}{rrrrr} & \% & \% & \% & \% \\ 1 & 22 & 30 & 44 & 4 \\ 2 & 7 & 43 & 27 & 23 \\ 3 & 12 & 45 & 25 & 18 \\ 4 & 17 & 28 & 38 & 17 \\ 5 & 6 & 24 & 32 & 38 \\ 6 & \text { nil } & 26 & 37 & 37\end{array}$

TABLE III. Lepromin results in patients with flat macules.

(a) Tests in Macules.

Group. Strongly positive. Positive. Doubtful. Negative

$\begin{array}{rrrrr} & \% & \% & \% & \% \\ 2 & 50 & 30 & -10 & 10 \\ 3 & 29 & 43 & 20 & 8 \\ 4 & 17 & 43 & 33 & 7 \\ 5 & 17 & 49 & 23 & 11 \\ 6 & 27 & 37 & 27 & 9\end{array}$

(b) Tests in normal skin.

Group. Strongly positive. Positive. Doubtful. Negative

$\begin{array}{lclll}1 & \% & \% & \% & \% \\ 2 & - & - & - & -10 \\ 3 & 4 & 90 & -42 & 28 \\ 4 & - & 26 & 40 & 34 \\ 5 & - & 31 & 38 & 31 \\ 6 & 9 & 27 & 50 & 18\end{array}$

Observations.

1. These results demonstrate that hypersensitiveness to lepromin is not the monopoly of optimal tuberculoid macules, but may be found in macules of every type, including lepromatous niacules.

2. The results of the test in macules are often in marker 
contrast with those in normal skin, especially at the lepromatous end of the series. The test in normal skin shows a progressive decline in positivity towards the lepromatous group, a finding in line with most published results. In macules, however, this tendency is very much less, strikingly so in raised macules. It foilow's that in macular leprosy hypersensitivity to lepromin may be localised, and may be exhibited at the site of infection even though it is not a property of normal skin.

3. In view of this allergy, it is desirable that records of lepromin tests should specify whether these have been performed in normal skin or not. Although it is a simple matter to select normal skin when macules are localised, this is far from being the case at the lepromatous end of the series, where widespread coalescence of macules may mask their existence.

4. In the raised macule series, the percentage of positive results remains between $77-90 \%$ throughout the series. This means that hypersensitivity to iepromin is not an infallible guide to the clinical outcome, for lepromatous macules, destined in many cases to degenerate into diffuse leproma, still gave positive results.

5. Results in raised macules are compared with those in flat macules in Table IV, in which figures from all groups are added together and expressed as percentages of the total.

TABLE IV. Comparison of raised with flat macules.

\begin{tabular}{lccccc} 
& \multicolumn{2}{c}{$\begin{array}{c}\text { Strongly positive. } \\
\%\end{array}$} & $\begin{array}{c}\text { Positive. } \\
\%\end{array}$ & $\begin{array}{c}\text { Doubtful. } \\
\%\end{array}$ & $\begin{array}{c}\text { Negative } \\
\%\end{array}$ \\
Raised macules & $\ldots \ldots \ldots .$. & 44 & 44 & 11 & 3 \\
Flat macules & $\ldots . . . . . . . .$. & 25 & 42 & 25 & 8
\end{tabular}

Results in flat macules are thus set at a lower level than in raised macules. Two factors may be responsible for this.

(a) Flat macules represent a milder response than raised macules, as histological studies of the optimal group indicate.

(b) Although throughout the series, efforts were made to select active cases, a proportion of flat macules were undoubtedly slightly raised macules in the process of resolution, and this additional factor may influence results.

6. It is necessary to notice that out of 385 macules tested, 22 were negative, and 64 gave a doubtful result. Of the 22 negative results, 8 were derived from the raised series, 14 from the flat series.

These observations accord with the characteristics of allergy in leprosy summarised in Part I, and it may be asserted with 
confidence that whatever other factors are involved, allergy is implicated in the production of macules of all types. In the leprous macule we see the visible resultant of the action of two major opposing forces : on the one hand the living leprosy bacillus, and on the other the defence mechanism of the skin, consisting of (a) the basic monocyte response, and (b) allergic hypersensitiveness with its antibody production and specific cellular reaction. Both sides in this conflict are inconstant, and both are influenced by secondary factors, and the macule is thus not a static form of the disease, but is dynamic, changing with variations in the factors responsible for it.

The dynamic nature of macules is well illustrated by the changes in type of macule which a single patient may exhibit from time to time. Recurrence in an old minor tuberculoid lesion may take the form of a spreading flat macule. An initial macule may be succeeded by a crop of macules ranging from major tuberculoid to lepromatous in type. The clinical appearance thus reflects the patient's immunological state at the moment only, and if the current response is unable to obliterate the infection, active macules of another type may later follow local spread of bacillary metastasis.

The effectiveness of the allergic response is determined by its timing and persistence rather than by its intensity. An optimal major tuberculoid response, far outweighing the bacillary force ranged against it, is usually fully effective, but it is not rare to see patients with highly elevated succulent lesions, anaesthetic, but scarcely hypopigmented, their edge poorly defined, in which bacilli are numerous and persistent. Such lesions may be strongly positive to lepromin, far more so than the surrounding skin. and the clinical outcome remains uncertain. Here it is apparent that the intensity of the response is not the decisive factor. It is indeed probable that its intensity and its persistence tend to vary inversely. This and similar questions can only be clarified by the observation of the lepromin test in macules of various types throughout their history, and work of this nature is continuing.

My thanks are due to $\dot{M}$ r. M. Smith, B.Sc. for technical assistance with lepromin tests, and to The Honourable Director of Medical Services, Nigeria, for permission to publish.

\section{REFERENCES.}

(1) Cairo Conference, reports of meetings, (1938). Internat. J. Lep. o, 392.

(2) Davey, T. F. (1946), Lep Rev. 17, No. 2.

(3) Davey, T. F. (1942), Lep. Rev. 13, 3.

(4) Dharmendra (1941), Lep. in Ind. 13, 77. 
(5) ERMakova, N. I. (1939), Inaternat. J. Lep. 7, 495.

(6) Lowe, J. (1936), Lep. in India, 8, No. 3.

(7) Rogers \& Muir (1940), Leprosy, 2nd Edition.

(8) Rogers \& Muir (1946), Leprosy, 3rd Edition.

(9) Wade, W. H. \& Ropriguez, J. N. (1937), Internat. J. Lep., 5, 1.

(10) Wade, W. H. \& Rodriguez, J. N. (1940), Internat. J. Lep., 8, 307. 